\title{
Heavy flavour decay properties with ATLAS
}

\author{
Ina Carli*t \\ Charles University, Faculty of Mathematics and Physics, \\ Institute of Particle and Nuclear Physics, \\ V Holesovickach 2, 18000 Prague, Czech Republic \\ E-mail: Ina.Carliecern.ch
}

\begin{abstract}
We present the results on CP-violation searches in the $B_{s}$ system, studied in the $B_{s} \rightarrow J / \psi \phi$ decay, and in the $B_{d}$ system through the comparison of the decay time distributions in the flavour specific state $J / \psi K^{*}$ and in the CP eigenstate $J / \psi K_{s}$. We also present new results in the search for the rare decays of $B_{d}$ and $B_{s}$ into $\mu^{+} \mu^{-}$. All results are based on the full sample of $p p$ collisions data recorded during LHC Run 1 by the ATLAS detector at $\sqrt{s} 7$ and $8 \mathrm{TeV}$. The consistency with the Standard Model predictions and with other available measurements is discussed.
\end{abstract}

Fourth Annual Large Hadron Collider Physics

13-18 June 2016

Lund, Sweden

* Speaker.

${ }^{\dagger}$ On behalf of the ATLAS Collaboration 


\section{Introduction}

The production of $B$ hadrons at the Large Hadron Collider [1] provides excellent opportunities for measurements of heavy flavour decay properties and indirect searches for new physics. Various rare decay modes occur in Standard Model (SM) at the loop level and are sensitive to contributions from physics phenomena beyond SM, mainly as a result of the interference with SM diagrams.

The ATLAS experiment [2] is a general-purpose detector with a cylindrical geometry and nearly $4 \pi$ coverage in solid angle. The Inner Detector (ID) detects tracks of charged particles and consists of pixel, silicon strip and transition radiation subdetectors embedded in a $2 \mathrm{~T}$ solenoidal magnetic field. The ID is surrounded by a liquid-argon sampling electromagnetic calorimeter and a steel/scintillator tile hadronic calorimeter. The outermost Muon Spectrometer (MS) consists of three large toroids and a system of gaseous detectors: monitored drift tubes and cathode strip chambers for precise measurements and resistive plate chambers and thin gap chambers for triggering.

The ATLAS trigger in LHC Run 1 was based on hardware Level-1 trigger and a 2-staged software High Level Trigger (HLT). The muon trigger at Level-1 is based on pattern search for different $p_{\mathrm{T}}$ thresholds in the MS. The regions of interest around these patterns are then used as seeds for the HLT reconstruction, matching information from both the MS and ID.

All results presented here are based on dataset collected during LHC Run 1 in 2011 (at $\sqrt{s}=$ $7 \mathrm{TeV}$ ) and 2012 (at $\sqrt{s}=8 \mathrm{TeV}$ ) corresponding to a total integrated luminosity of $25 \mathrm{fb}^{-1}$.

\section{Measurement of the CP-violating phase $\phi_{s}$ and $B_{s}$ decay width difference $\Delta \Gamma_{s}$ with $B_{s} \rightarrow J / \psi \phi$ decay}

New phenomena beyond SM may alter the CP-violation in decays of $B$ hadrons. The $B_{s} \rightarrow$ $J / \psi \phi$ decay is expected to be sensitive to these contributions because the CP-violation emerges from interference between direct decays and $B_{s}-\overline{B_{s}}$ mixing. The CP-violating phase $\phi_{s}$ is predicted to be small in SM [3] and is a good candidate for new physics searches.

Events for this measurement [4] are selected by di-muon triggers searching for $J / \psi \rightarrow \mu^{+} \mu^{-}$ candidates with muon $p_{\mathrm{T}}$ thresholds of $4 \mathrm{GeV}$ or $6 \mathrm{GeV}$. The muon object is identified either as a combination of ID and MS track segments, referred to as a combined muon, or segment-tagged muon, formed from a MS track segment not associated with MS track but matched to an ID track. Muon track parameters are determined by the ID measurement only, since the precision of track parameters is dominated by the ID track reconstruction in the kinematic range of this analysis. The $\phi \rightarrow K^{+} K^{-}$candidates are reconstructed from tracks with $p_{\mathrm{T}}>1 \mathrm{GeV}$ and $|\eta|<2.5$ not identified as muons and each combination of $J / \psi$ and $\phi$ is fitted to a common vertex. Candidates are accepted if the invariant mass of hadronic track pairs falls within interval $\left(1.0085 \mathrm{GeV}<m_{K^{+} K^{-}}<1.0305\right.$ $\mathrm{GeV}$ ) around $\phi$ mass and if the criteria on vertex fit quality and number of ID hits for tracks are met. In total, $376987 B_{s}$ candidates are selected in $B_{s}$ mass range of $5.15-5.65 \mathrm{GeV}$.

The initial flavour of $B_{s}$ meson is measured by opposite-side tagging (OST) which is extracting information from the second $b$-quark present in the event. Several tagging methods are implemented: the measured charge of a muon or electron from a semileptonic decay of $B$ meson provides a strong separation power but the result is diluted because of neutral $B$ meson oscillations and cascade decays $b \rightarrow c \rightarrow \ell$. For muon-based tagging, methods using combined or segment-tagged 


\begin{tabular}{l|c|c|c}
\hline Tagger & Efficiency [\%] & Dilution [\%] & Tagging Power [\%] \\
\hline \hline Combined $\mu$ & $4.12 \pm 0.02$ & $47.4 \pm 0.2$ & $0.92 \pm 0.02$ \\
Electron & $1.19 \pm 0.01$ & $49.2 \pm 0.3$ & $0.29 \pm 0.01$ \\
Segment-tagged $\mu$ & $1.20 \pm 0.01$ & $28.6 \pm 0.2$ & $0.10 \pm 0.01$ \\
Jet-charge & $13.15 \pm 0.03$ & $11.85 \pm 0.03$ & $0.19 \pm 0.01$ \\
\hline Total & $19.66 \pm 0.04$ & $27.56 \pm 0.06$ & $1.49 \pm 0.02$ \\
\hline
\end{tabular}

Table 1: Summary of tagging performance for the different flavour tagging methods [4]. Uncertainties shown are statistical only. The efficiency and tagging power are each determined by summing over the individual bins of the charge distribution. The effective dilution is obtained from the measured efficiency and tagging power. For the efficiency, dilution, and tagging power, the corresponding uncertainty is determined by combining the appropriate uncertainties in the individual bins of each charge distribution.

muons are treated as distinct and are optimised separately. For electron-based tagging, an electron is identified using information from the ID and is required to satisfy tight electron quality criteria and kinematic cuts to remove electrons from other vertices and from signal-side of the decay. If more than one lepton is found in the event, the lepton with the highest $p_{\mathrm{T}}$ is selected. A lepton cone charge is measured as a weighted sum of the charge of tracks in a cone $\Delta R<0.5$ around the lepton direction. If no lepton is present, a weighted sum of the charge of tracks in a jet tagged by b-tagging is used. To study the OST for this analysis, a sample of self-tagging $B^{ \pm} \rightarrow J / \psi K^{ \pm}$was selected from 2012 dataset satisfying similar selection as signal candidates. Tagging performance of all flavour tagging methods is summarized in Table 1.

An unbinned maximum likelihood fit is performed on selected events to extract parameters of the signal decay. The fit uses reconstructed mass, proper decay time, proper decay time uncertainty, tagging probability and transversity angles of each candidate. The likelihood function is defined as a combination of probability density functions $\mathscr{F}_{\mathrm{s}}$ for signal, $\mathscr{F}_{B^{0}}$ and $\mathscr{F}_{\Lambda_{b}}$ for $B^{0}$ and $\Lambda_{b}$ background contributions and $\mathscr{F}_{\text {bkg }}$ for other backgrounds as

$$
\ln \mathscr{L}=\sum_{i=1}^{N}\left\{w_{i} \cdot \ln \left(f_{\mathrm{s}} \mathscr{F}_{\mathrm{s}}+f_{\mathrm{s}} f_{B^{0}} \mathscr{F}_{B^{0}}+f_{\mathrm{s}} f_{\Lambda_{b}} \mathscr{F}_{\Lambda_{b}}+\left(1-f_{\mathrm{s}}\left(1+f_{B^{0}}+f_{\Lambda_{b}}\right)\right) \mathscr{F}_{\mathrm{bkg}}\right)\right\}
$$

where $N$ is the number of candidates, $w_{i}$ is the trigger weight, $f_{s}$ is the fraction of signal candidates and $f_{B^{0}}\left(f_{\Lambda_{b}}\right)$ are the fractions of $B^{0}\left(\Lambda_{b}\right)$ events misidentified as $B_{s}$. The full fit depends on 9 physical parameters and additional parameters such as signal and background fractions, parameters describing mass, proper decay time, flavour and angular distributions of signal and background events, as well as the detector acceptance. In total, the fit extracted $74900 \pm 400$ signal candidates. The mass, proper decay time and angle fit projections can be seen in Figures 1 and 2.

Figure 3 shows the likelihood contours in $\phi_{s}-\Delta \Gamma_{s}$ plane, together with the SM expectations. Measured values are consistent with those obtained in the previous analysis of $7 \mathrm{TeV}$ data. A Best Linear Unbiased Estimate combination [5] is used to combine the $7 \mathrm{TeV}$ and $8 \mathrm{TeV}$ measurements. 

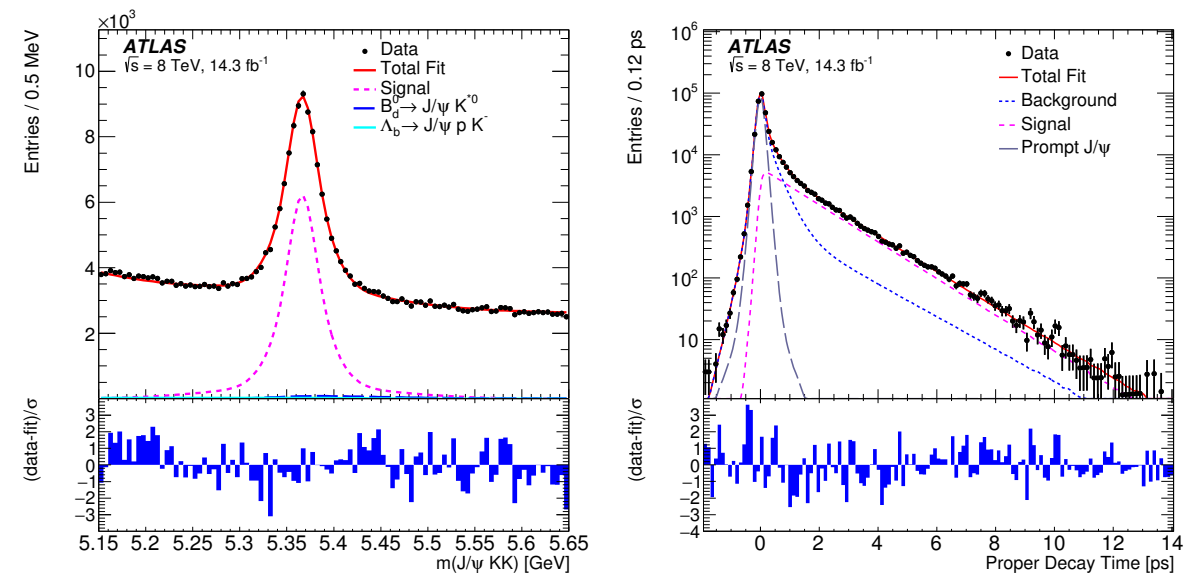

Figure 1: Mass and proper decay time fit projections for the $B_{s} \rightarrow J / \psi \phi$ sample. [4]
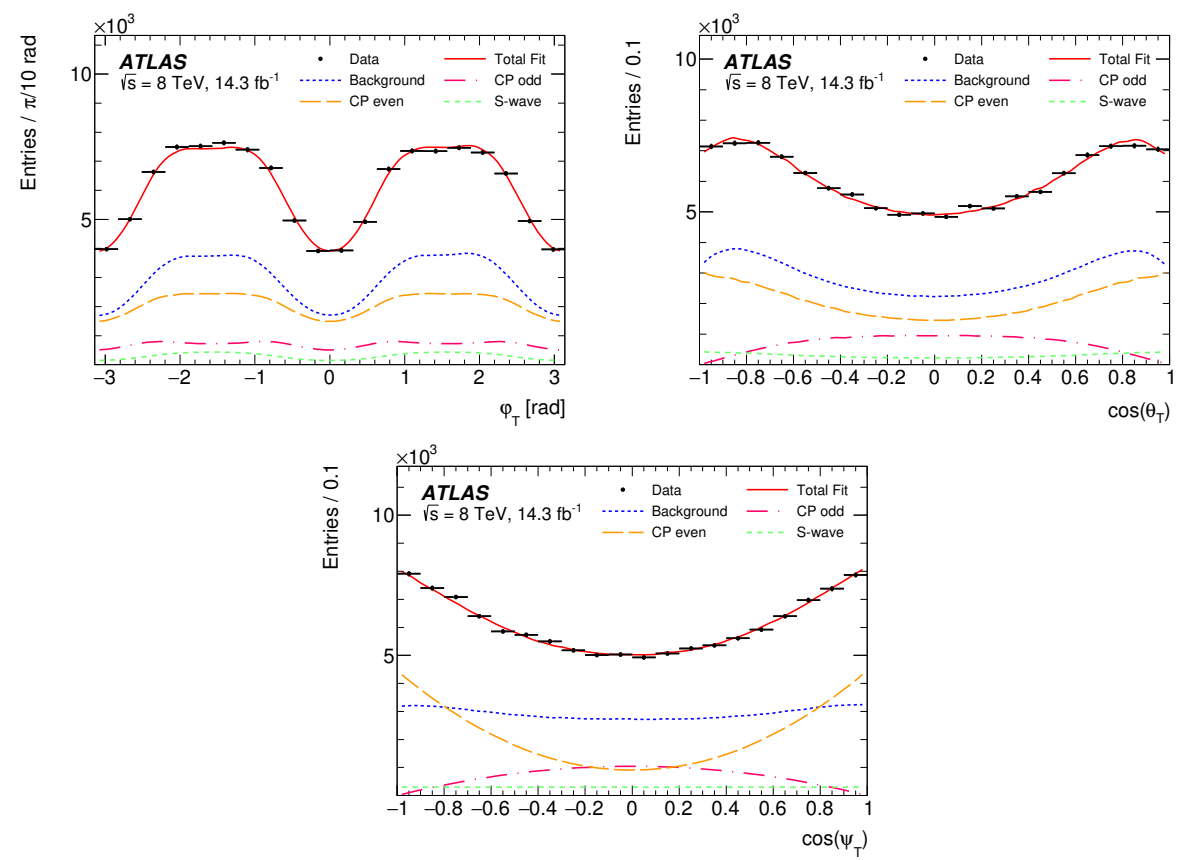

Figure 2: Fit projections of the transversity angles for the $B_{s} \rightarrow J / \psi \phi$ sample. [4]

The combined results for fit parameters are [4]

$$
\begin{aligned}
\phi_{s} & =-0.090 \pm 0.078 \text { (stat.) } \pm 0.041 \text { (syst.) } \mathrm{rad} \\
\Delta \Gamma_{s} & =0.085 \pm 0.011 \text { (stat.) } \pm 0.007 \text { (syst.) } \mathrm{ps}^{-1} \\
\Gamma_{s} & =0.675 \pm 0.003 \text { (stat.) } \pm 0.003 \text { (syst.) } \mathrm{ps}^{-1} \\
\left|A_{\|}(0)\right|^{2} & =0.227 \pm 0.004 \text { (stat.) } \pm 0.006 \text { (syst.) } \\
\left|A_{0}(0)\right|^{2}= & 0.522 \pm 0.003 \text { (stat.) } \pm 0.007 \text { (syst.) } \\
\left|A_{S}(0)\right|^{2} & =0.072 \pm 0.007 \text { (stat.) } \pm 0.018 \text { (syst.) } \\
\delta_{\perp}= & 4.15 \pm 0.32 \text { (stat.) } \pm 0.16 \text { (syst.) rad } \\
\delta_{\|}= & 3.15 \pm 0.10 \text { (stat.) } \pm 0.05 \text { (syst.) rad } \\
\delta_{\perp}-\delta_{S}= & -0.08 \pm 0.03 \text { (stat.) } \pm 0.01 \text { (syst.) rad. }
\end{aligned}
$$


The likelihood contours in the $\phi_{s}-\Delta \Gamma_{s}$ plane for the combination are shown in Figure 3 (right). They agree with the SM predictions.
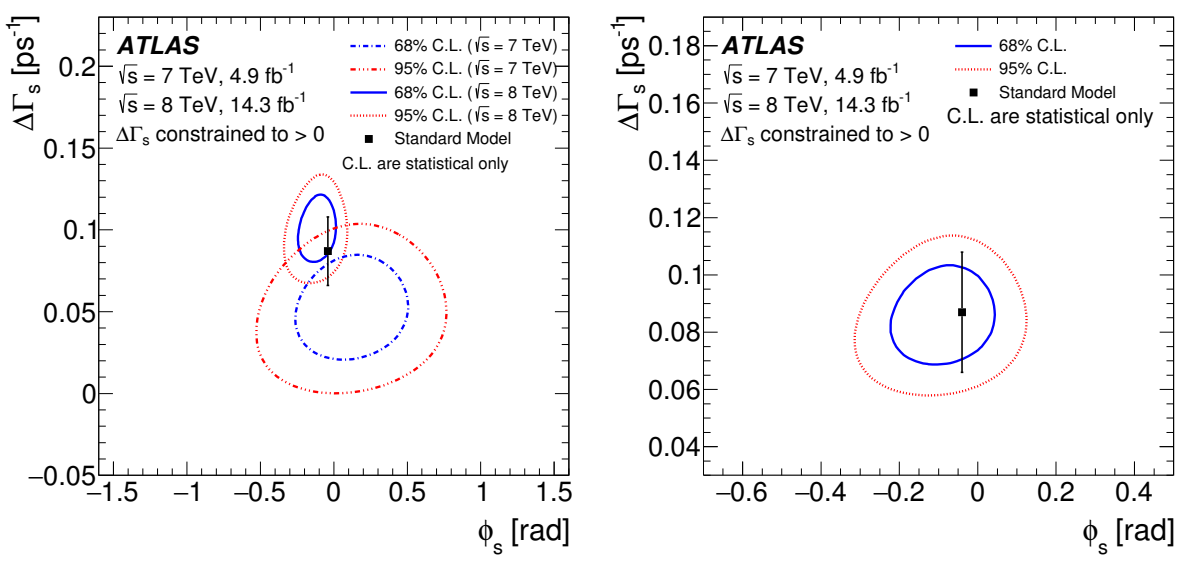

Figure 3: Likelihood contours in the $\phi_{s}-\Delta \Gamma_{s}$ plane for individual results from $7 \mathrm{TeV}$ and $8 \mathrm{TeV}$ data (left) and a final statistical combination of the results from $7 \mathrm{TeV}$ and $8 \mathrm{TeV}$ data (right). [4]

\section{Study of the rare decays of $B_{d}$ and $B_{s}$ into muon pairs}

Indirect searches for physics beyond the SM include decays mediated by flavour-changing neutral currents that are highly suppressed in the SM. The branching fractions of rare decays $B_{(s)} \rightarrow$ $\mu^{+} \mu^{-}$are of particular interest because of the additional helicity suppression.

The $B_{(s)} \rightarrow \mu^{+} \mu^{-}$branching fractions are measured as a relative branching ratio with a reference decay $B^{ \pm} \rightarrow J / \psi\left(\rightarrow \mu^{+} \mu^{-}\right) K^{ \pm}$in order to minimise systematic uncertainties [6]. A dimuon trigger was used to select events and the 2011 sample contains events with two muons with $p_{\mathrm{T}}>$ $4 \mathrm{GeV}$. Due to higher pile-up during the 2012 data taking, this trigger was prescaled in the beginning of each run so a tighter selection was applied: $p_{\mathrm{T}}>6 \mathrm{GeV}$ or $|\eta|<1.05$ for one of the muons. Additional loose cut on dimuon invariant mass was applied at the HLT level to select events in the $B_{(s)}$ or $J / \psi$ mass window. After offline reconstruction, a preselection is made requiring muons with $p_{\mathrm{T}}>4 \mathrm{GeV}$, good quality of tracks and reconstructed vertices and minimal numbers of hits in the ID subdetectors. Similar cuts are applied to reference channel candidates with $p_{\mathrm{T}}>1 \mathrm{GeV}$ for kaons. This procedure selected $2.6 \times 10^{6}$ signal and $2.3 \times 10^{6}$ reference channel candidates in the signal regions (for the $B_{(s)}$ the signal region is defined as $5166 \mathrm{MeV}<m_{\mu^{+} \mu^{-}}<5526 \mathrm{MeV}$ ).

Three categories of background events are considered: the combinatorial background is studied in signal mass sidebands and it is found to be correctly described by the inclusive $b \bar{b} \rightarrow \mu \mu X$ MC sample. A boosted decision tree (BDT) based on 15 variables describing $B$ meson vertex, muons forming the candidate and variables related to the rest of event, is used to reduce this component. The resulting signal efficiency of continuum-BDT is $54 \%$ with a reduction of combinatorial background by a factor of about $10^{3}$. The second source is formed by partially reconstructed $B \rightarrow \mu \mu X$ decays that are characterized by non-reconstructed particles and thus are accumulating in the low-mass sideband. Several topologies contribute to this component: same-side background from decay cascades $b \rightarrow c \mu \rightarrow s(d) \mu \mu$, same vertex background from $B$ decays containing a 

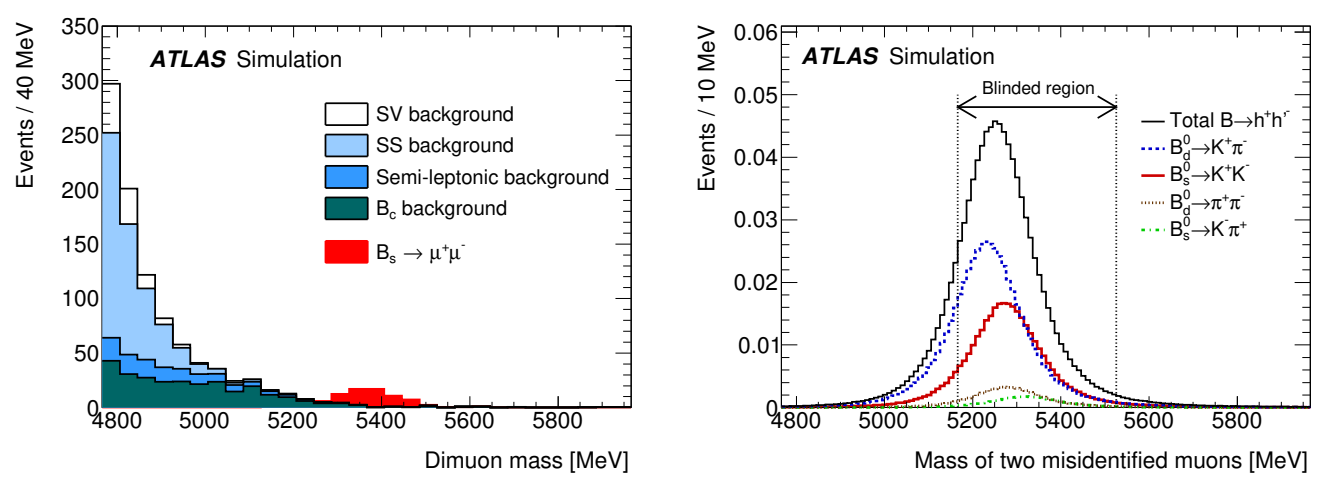

Figure 4: Dimuon mass distribution for the partially reconstructed background from simulation, together with the SM expectation for the $B_{s} \rightarrow \mu^{+} \mu^{-}$signal (left), and mass distribution of the peaking background components from $B_{s} \rightarrow h h^{\prime}$ (right). All distributions are normalised to the expected yield for the analysed integrated luminosity. [6]

muon pair $\left(B \rightarrow K^{*} \mu^{+} \mu^{-}, B \rightarrow J / \psi X \rightarrow \mu \mu \mu X^{\prime}\right), B_{c}$ decays (e.g. $\left.B_{c} \rightarrow J / \psi \mu \rightarrow \mu \mu \mu\right)$ and semileptonic $B$ decays where the final-state hadron is misidentified as muon (mainly $B \rightarrow \pi \mu \nu$, $B_{s} \rightarrow K \mu \nu$ or $\left.\Lambda_{b} \rightarrow p \mu v\right)$. Inclusive MC samples were generated and their dimuon mass distributions are shown in Figure 4 (left). Finally, the peaking background due to $B_{(s)}$ decays with two hadrons misidentified as muons contributes to the signal region, as can be seen in Figure 4 (right). After preselection the probability for a kaon (pion) to be misidentified as muon is $0.4 \%(0.2 \%)$, the probability for protons is negligible $(<0.01 \%)$. An additional BDT is tuned for $95 \%$ efficiency for the signal sample while it reduces the hadron misidentification by a factor of 0.37 , resulting in misidentification probability of $0.09 \%$ for kaons and $0.04 \%$ for pions.

The candidates passing preselection are classified to three intervals in the continuum-BDT with equal efficiency for signal events and an unbinned maximum-likelihood fit of dimuon invariant mass is performed. Figure 5 shows the dimuon mass in three intervals of the continuum-BDT with results of the fit. Without applying any boundary on fitted values, the number of signal $B_{s}$ and $B_{d}$ candidates is $N_{s}=16 \pm 12$ and $N_{d}=-11 \pm 9$. Restricting yields to be non-negative, the resulting values are $N_{s}=11$ and $N_{d}=0$.

The branching fractions for $B_{s} \rightarrow \mu^{+} \mu^{-}$and $B_{d} \rightarrow \mu^{+} \mu^{-}$are extracted from data using a profile-likelihood fit. The value of relative hadronisation probability $f_{s} / f_{d}$ used in normalisation terms is taken from the ATLAS measurement $f_{s} / f_{d}=0.240 \pm 0.020$ [7], assuming $f_{u} / f_{d}=1$.

Figure 6 shows the fit result as likelihood contours in plane of $\mathscr{B}\left(B_{s} \rightarrow \mu^{+} \mu^{-}\right)-\mathscr{B}\left(B_{d} \rightarrow\right.$ $\mu^{+} \mu^{-}$) allowing negative values of branching fractions. Using the $\mathrm{CL}_{\mathrm{S}}$ method [8], upper limits are placed on the branching ratios

$$
\begin{aligned}
\mathscr{B}\left(B_{d} \rightarrow \mu^{+} \mu^{-}\right) & <4.2 \times 10^{-10} 95 \% \mathrm{CL} \\
\mathscr{B}\left(B_{s} \rightarrow \mu^{+} \mu^{-}\right) & <3.0 \times 10^{-9} 95 \% \mathrm{CL} .
\end{aligned}
$$

A Neyman construction [9] is used to determine the $68.3 \%$ confidence interval for $\mathscr{B}\left(B_{s} \rightarrow \mu^{+} \mu^{-}\right)$ with pseudo-MC experiments as

$$
\mathscr{B}\left(B_{s} \rightarrow \mu^{+} \mu^{-}\right)=\left(0.9_{-0.8}^{+1.1}\right) \times 10^{-9} .
$$



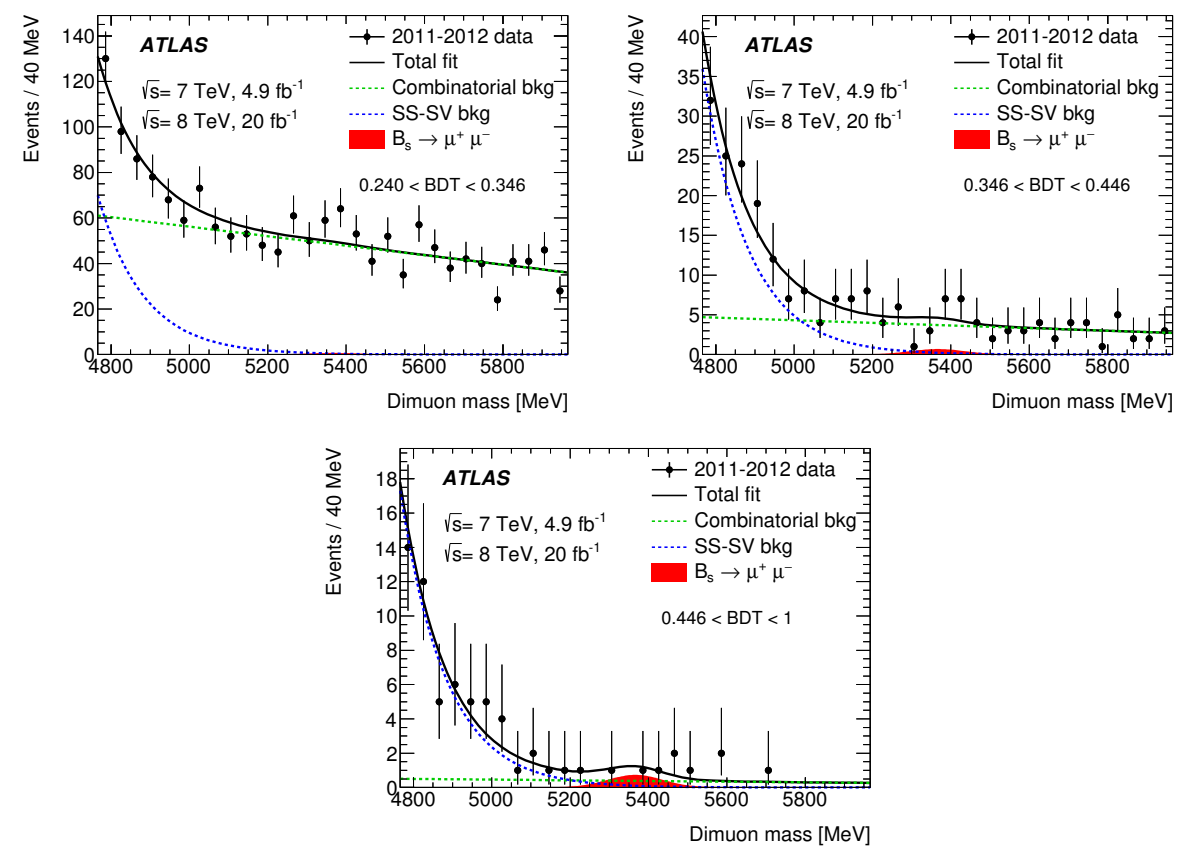

Figure 5: Dimuon invariant mass distributions in three intervals of continuum-BDT. Superimposed is the result of the maximum-likelihood fit, obtained imposing the boundary of non-negative signal yields. [6]

The $\mathscr{B}\left(B_{d} \rightarrow \mu^{+} \mu^{-}\right)$limit is compatible with predictions based on the SM and with the combined result of CMS and LHCb experiments. The upper limit on $\mathscr{B}\left(B_{s} \rightarrow \mu^{+} \mu^{-}\right)$is lower than SM prediction and in better agreement with the measurement of CMS and LHCb.

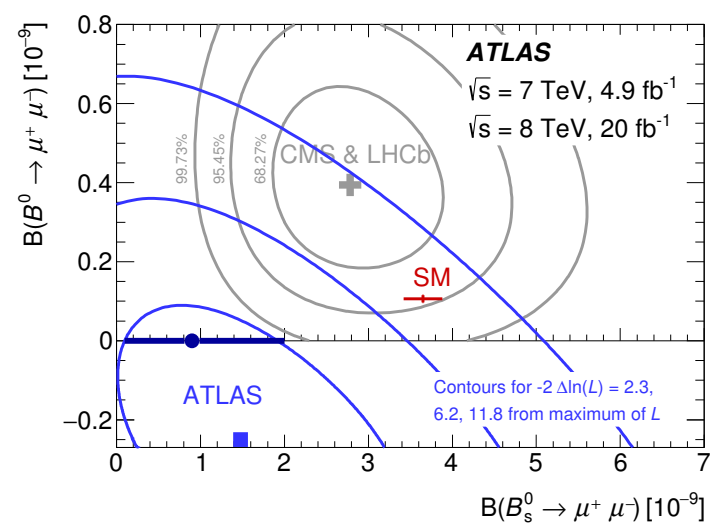

Figure 6: Likelihood contours in plane of $\mathscr{B}\left(B_{s} \rightarrow \mu^{+} \mu^{-}\right)-\mathscr{B}\left(B_{d} \rightarrow \mu^{+} \mu^{-}\right)$for intervals of $-2 \Delta \ln (L)$ equal to $2.3,6.2$ and 11.8 of maximum likelihood allowing negative values of the branching fractions. The maximum within physical boundary is shown with error bars for $68.3 \%$ interval for $\mathscr{B}\left(B_{s} \rightarrow \mu^{+} \mu^{-}\right)$. Results of the CMS and LHCb combination and SM predictions are also shown. [6] 


\section{Measurement of relative width difference of the $B_{d}-\overline{B_{d}}$ system}

The width difference $\Delta \Gamma_{d}$ describes time evolution of the $B_{d}-\overline{B_{d}}$ system and the relative value $\Delta \Gamma_{d} / \Gamma_{d}$ is predicted to be $(0.42 \pm 0.08) \times 10^{-2}$ in the SM, while the experimental uncertainty is large, $1 \times 10^{-2}$ [3]. The ATLAS analysis [10] is based on measurement of the ratio of the time evolution of $B_{d} \rightarrow J / \psi K_{\mathrm{S}}$ and $B_{d} \rightarrow J / \psi K^{*}$ decays. The decay rates can be described as

$$
\begin{aligned}
B_{d} \rightarrow J / \psi K_{\mathrm{S}}: \Gamma_{d}(t) & \sim e^{-\Gamma_{d} t}\left(\cosh \frac{\Delta \Gamma_{d} t}{2}+\cos (2 \beta) \sinh \frac{\Delta \Gamma_{d} t}{2}-A_{\mathrm{P}} \sin (2 \beta) \sin \left(\Delta m_{d} t\right)\right) \\
B_{d} \rightarrow J / \psi K^{*}: \Gamma_{d}(t) & \sim e^{-\Gamma t} \cosh \frac{\Delta \Gamma_{d} t}{2}
\end{aligned}
$$

where $t$ is the proper decay time of $B_{d}$ meson, $\beta$ is the angle of the unitarity triangle of CKM matrix, $A_{\mathrm{P}}$ is the production asymmetry of $B_{d}$ and $\overline{B_{d}}$ in $p p$ collisions and $\Delta m_{d}$ is the mass difference of physical mass eigenstates. In this analysis, the proper decay length of the $B_{d}$ meson, $L_{\text {prop }}^{B}=c t$, is used in place of the proper decay time $t$. The width difference $\Delta \Gamma_{d}$ can be than obtained from fit to the ratio $R\left(L_{\text {prop }}^{B}\right)$ of the distributions of the number of reconstructed $B_{d} \rightarrow J / \psi K_{\mathrm{S}}$ and $B_{d} \rightarrow J / \psi K^{*}$ decays which cancel many experimental uncertainties.

To select events for analysis, a set of dimuon trigger chains for $J / \psi \rightarrow \mu^{+} \mu^{-}$was used. During offline reconstruction, the $J / \psi$ candidates are formed from pairs of oppositely charged muons with $p_{\mathrm{T}}>2.5 \mathrm{GeV}$ and $|\eta|<2.4$. The $K_{\mathrm{S}} \rightarrow \pi^{+} \pi^{-}$and $K^{*} \rightarrow K^{+} \pi^{-}$candidates are reconstructed from pairs of oppositely charged tracks with $p_{\mathrm{T}}>400 \mathrm{MeV}$ (or $800 \mathrm{MeV}$ for kaon track from $K^{*}$ ). Additional criteria on number of ID tracks, fit quality of all vertices and projection of the distance between the $J / \psi$ and $K_{\mathrm{S}}\left(K^{*}\right)$ vertices are applied. The masses of $K_{\mathrm{S}}$ and $K^{*}$ candidate are required to be in (450-550) MeV and (850-950) MeV intervals. No particle identification is used for the reconstruction of the $K^{*}$. Therefore each combination of mass hypotheses is assigned to the hadron tracks and the combination with smaller deviation from the nominal $K^{*}$ mass is used.

The total number of $B_{d} \rightarrow J / \psi K_{\mathrm{S}}$ decays extracted from fit is $28170 \pm 250$ for the 2011 dataset and $110830 \pm 520$ for the 2012 dataset. For $B_{d} \rightarrow J / \psi K^{*}$ decays, the corresponding values are $129200 \pm 900$ and $555800 \pm 1900$ candidates, respectively. Figure 7 shows example of the fitted mass distributions for decay length bin $(0-0.3) \mathrm{mm}$.
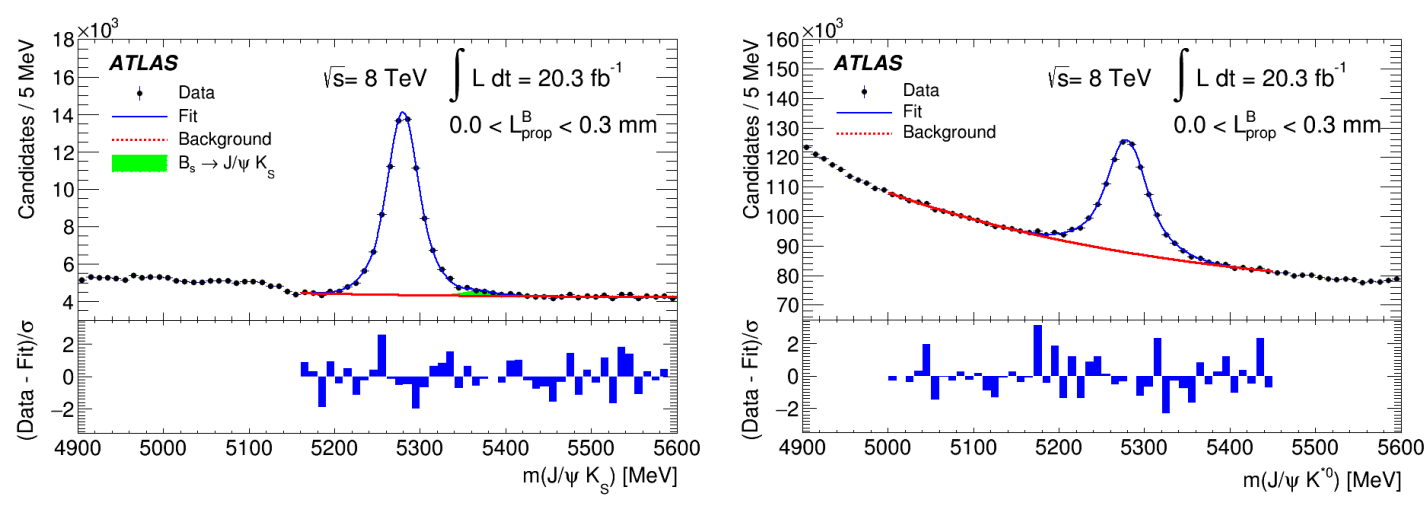

Figure 7: The invariant mass distributions of the $B_{d} \rightarrow J / \psi K_{\mathrm{S}}$ candidates (left) and $B_{d} \rightarrow$ $J / \psi K^{*}$ candidates (right) for the 2012 data sample for $0.0<L_{\text {prop }}^{B}<0.3 \mathrm{~mm}$. [10] 
The production asymmetry $A_{\mathrm{P}}$ of the $B_{d}$ mesons can be obtained from the time-dependent charge asymmetry of the flavour-specific $B_{d} \rightarrow J / \psi K^{*}$ and it's anti-decay. In addition to the oscillations, the differences in reconstruction of positive and negative particle tracks introduces a detector-related asymmetry $A_{\text {det }}$ (mainly from a different interaction cross-section of charged kaons with the ID material). Asymmetries are further diluted by misidentification of pion and kaon from the $K^{*}$. The detector and production asymmetries are measured to be

$$
\begin{aligned}
A_{\text {det }} & =(1.33 \pm 0.24 \text { (stat. }) \pm 0.30 \text { (syst. })) \times 10^{-2} \\
A_{\text {prod }} & =(0.25 \pm 0.48 \text { (stat. }) \pm 0.05 \text { (syst. })) \times 10^{-2} .
\end{aligned}
$$
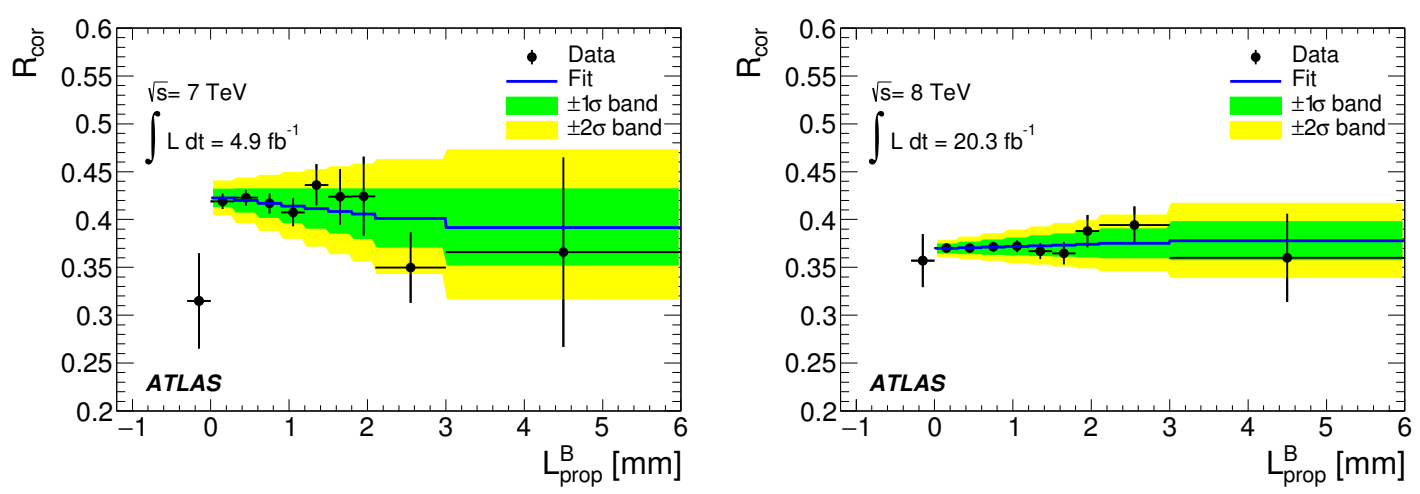

Figure 8: Efficiency-corrected ratio of the observed decay length distributions, $R_{\text {corr }}\left(L_{\text {prop }}^{B}\right)$ for the 2011 (left) and the 2012 (right) datasets. The normalisation of the two datasets is arbitrary. [10]

Finally, the ratio of observed events is corrected for efficiency and the resulting distribution is fitted in bins of $L_{\text {prop }}^{B}$ separately for the 2011 and the 2012 samples. The results of the fit are shown in Figure 8 and the fitted values are

$$
\begin{aligned}
& \left.\Delta \Gamma_{d} / \Gamma_{d}=(-2.8 \pm 2.2 \text { (stat.) } \pm 1.7 \text { (syst. })\right) \times 10^{-2}(7 \mathrm{TeV}) \\
& \left.\Delta \Gamma_{d} / \Gamma_{d}=(0.8 \pm 1.3 \text { (stat.) } \pm 0.8 \text { (syst. })\right) \times 10^{-2}(8 \mathrm{TeV}) \\
& \left.\left.\Delta \Gamma_{d} / \Gamma_{d}=(-0.1 \pm 1.1 \text { (stat. }) \pm 0.9 \text { (syst. }\right)\right) \times 10^{-2}(\text { combined })
\end{aligned}
$$

where the combination is done using $\chi^{2}$ method. It is currently the most precise measurement of this quantity. It agrees well with the SM prediction and it is consistent with measurements done by other experiments.

\section{Conclusions}

Analyses of rare $B$-hadron decays using dataset collected during Run 1 are presented. No deviations from the Standard model predictios are found and the results are consistent with measurements done by other experiments.

\section{Acknowledgments}

The author acknowledges support of grant LG15052 of Ministry of Education, Youth and Sports of the Czech Republic. 


\section{References}

[1] L. Evans and P. Bryant (editors), LHC Machine, JINST 3 S08001 (2008).

[2] ATLAS Collaboration, The ATLAS Experiment at the CERN Large Hadron Collider, JINST 3, S08003 (2008).

[3] Particle Data Group Collaboration, K. A. Olive et al., Review of Particle Physics, Chin. Phys. C 38 (2014) 090001.

[4] ATLAS Collaboration, Measurement of the CP-violating phase $\phi_{s}$ and the $B_{s}^{0}$ meson decay width difference with $B_{s}^{0} \rightarrow J / \psi \phi$ decays in ATLAS, JHEP 08 (2016) 147.

[5] R. Nisius, On the combination of correlated estimates of a physics observable, Eur. Phys. J. C 74 (2014) 1-20.

[6] ATLAS Collaboration, Study of the rare decays $B_{s}^{0}$ and $B^{0}$ into muon pairs from data collected during the LHC Run 1 with the ATLAS detector, arXiv:1604.04263[hep-ex].

[7] ATLAS Collaboration, Determination of the ratio of b-quark fragmentation fractions $f_{s} / f_{d}$ in $p p$ collisions at $\sqrt{s}=7$ TeV with the ATLAS Detector, Phys. Rev. Lett. 115 (2015) 262001, arXiv:1507.08925 [hep-ex].

[8] A. L. Read, Presentation of search results: The $C L_{s}$ technique, J. Phys. G 28 (2002) 2693-2704.

[9] J. Neyman, Outline of a Theory of Statistical Estimation Based on the Classical Theory of Probability, Phil. Trans. R. Soc. London A, 236 (1937) 333-380.

[10] ATLAS Collaboration, Measurement of the relative width difference of the $B^{0}-\bar{B}^{0}$ system with the ATLAS detector, JHEP 06 (2016) 081, arXiv:1605.07485[hep-ex]. 